\title{
Role of Smad3 signaling in the epithelial-mesenchymal transition of the lens epithelium following injury
}

\author{
FANLAN MENG ${ }^{1,2}$, JUN LI $^{1,2}$, XIAO YANG $^{3}$, XIAOYONG YUAN $^{1,2}$ and XIN TANG ${ }^{1,2}$ \\ ${ }^{1}$ Clinical College of Ophthalmology, Tianjin Medical University; ${ }^{2}$ Tianjin Eye Hospital, Tianjin Key Laboratory of \\ Ophthalmology and Visual Science, Tianjin Eye Institute, Tianjin 300020; ${ }^{3}$ The State Key Laboratory of Proteomics, \\ Genetics Laboratory of Development and Disease, Institute of Biotechnology, AMMS, Beijing 100071, P.R. China
}

Received November 22, 2017; Accepted May 4, 2018

DOI: $10.3892 /$ ijmm.2018.3662

\begin{abstract}
Transforming growth factor- $\beta$ (TGF- $\beta$ ) is important in the development of posterior capsule opacification (PCO), and inhibition of the TGF- $\beta$ pathway may represent a novel method of treating PCO. Drosophila protein, mothers against decapentaplegic homolog $3(\operatorname{Smad} 3)$ is a phosphorylated receptor-activated Smad required for the transmission of TGF- $\beta$ signals. Smad3 knockout (KO) disturbs the activation of TGF- $\beta$ signaling, thus inhibiting the onset of PCO. In the present study, lens epithelial cell (LEC) damage induced by extracapsular cataract extraction was simulated by puncture of the anterior capsule using a 26-gauge hypodermic needle. The effect of Smad3 in the trauma-induced epithelial-mesenchymal transition (EMT) of the lens epithelium in Smad3-KO and wild-type (WT) mice was then observed. The expression levels of EMT markers and extracellular matrix components were measured in the two groups by reverse transcription-quantitative polymerase chain reaction analysis, western blot analysis and immunofluorescence staining. Apoptosis was also detected in the punctured anterior capsule. The Smad3-KO mice exhibited lower expression levels of $\alpha$-smooth muscle actin, lumican, osteopontin, fibronectin and collagen, compared with the WT mice. Additionally, the Smad3-KO mice exhibited a higher percentage of apoptotic cells than the WT mice. Smad3 signaling was associated with the induction of trauma-induced EMT, and Smad3 KO interfered with TGF- $\beta$ signaling pathway activation, but did not completely inhibit the trauma-induced EMT in LECs.
\end{abstract}

Correspondence to: Dr Xiaoyong Yuan or Professor Xin Tang, Tianjin Eye Hospital, Tianjin Key Laboratory of Ophthalmology and Visual Science, Tianjin Eye Institute, 4 Gansu Road, Heping, Tianjin 300020, P.R. China

E-mail: yuanxy_cn@hotmail.com

E-mail: tangxin_eye@163.com

Key words: posterior capsule opacification, mothers against decapentaplegic homolog 3 (Drosophila) knockout, lens epithelial cells, transforming growth factor- $\beta$, epithelial-mesenchymal transition
Therefore, Smad3 may be a target in the treatment of PCO and other fibrosis-related diseases.

\section{Introduction}

Posterior capsule opacification (PCO) is a secondary cataract, which is the most important complication of extracapsular cataract extraction and the main reason for postoperative visual decline (1). The main molecular biological mechanism underlying the development of PCO involves the transformation of residual lens epithelial cells (LECs) into mesenchymal cells and the migration of these cells to the posterior capsule, leading to abnormal extracellular matrix (ECM) deposition on and fibrosis of the posterior capsule of the lens (1-4).

The expression of transforming growth factor (TGF) is elevated in the anterior chamber following cataract surgery, and TGF- $\beta$ is currently considered one of the most important promoters of LEC transdifferentiation, which leads to pathological fibrosis (5). During the epithelial-mesenchymal transition (EMT), LECs transform into myofibroblasts and undergo cytoskeletal rearrangements, with events associated with the deposition of several ECM proteins, including lumican, collagen, fibronectin, hyaluronic acid (HA) (6) and osteopontin (OPN) (7). It has been reported that TGF- $\beta$ regulates numerous cell biological behaviors by activating mothers against decapentaplegic homolog (Smad)-mediated signal transduction pathways (8). Smad2 and Smad3 are phosphorylated receptor-activated Smads, which are essential for the transmission of TGF- $\beta$ signals. However, Smad2 and Smad3 have different biological functions. Smad2 knockout (KO) causes mice to die before they are born, whereas Smad3-KO mice survive until birth but exhibit mucosal immunodeficiency (9). Granulation tissue lacking Smad3 produces limited quantities of ECM (10). Additionally, the loss of Smad3 disturbs the TGF- $\beta$-mediated induction of the EMT process and renal fibrosis $(11,12)$.

Smad3, an integral part of the TGF- $\beta / \mathrm{Smad} 3$ signaling pathway, is closely associated with fibrotic diseases and the TGF- $\beta 1 / \mathrm{Smad} 3$ pathway is involved in renal fibrosis in patients with kidney disease (13). Smad3 $\mathrm{KO}\left(\mathrm{Smad}^{-/}\right)$ was found to reduce esophageal fibrosis in a model of egg-induced eosinophilic esophagitis (14). Skin cells derived from Smad3-KO mice show less severe inflammation and 
enhanced wound healing (15). According to the results of our previous in vitro experiments, silencing of Smad3 interfered with TGF- $\beta 2$-induced cell proliferation and downstream ECM protein (10). In addition, the overexpression of Smad3 was found to activate the TGF- $\beta 2$ signaling pathway and increased the secretion of ECM components in the human lens epithelial (HLE)-B3 human lens epithelial cell line (16).

The present study investigated whether Smad3 KO prevented trauma-induced EMT of the lens epithelium in vivo. In addition, the study investigated whether inhibition of the phosphorylation of Smad3 (p-Smad) by SIS3 affected ECM production in vitro. The potential association between apoptosis and Smad3 was also determined. Wild-type $\left(\operatorname{Smad}_{3} 3^{++}\right.$; WT) and Smad3 $3^{--}$mice were used to reveal the role of Smad3 in the lens epithelium following injury. The mRNA or protein expression levels of multiple EMT markers, including $\alpha$-smooth muscle actin ( $\alpha$-SMA), Snaill, Slug (Snail 2), Twist1, SMAD-interacting protein 1 (SIP1), E-cadherin, lumican, fibronectin, OPN, HA and collagen, were measured. Inhibition of the TGF- $\beta /$ Smad 3 pathway may be clinically desirable for the prevention of capsular opacification, a potential complication of cataract surgery.

\section{Materials and methods}

Animals and traumatic cataract model generation. All experimental procedures were performed according to the ARVO Declaration on the Use of Animals in Ophthalmic and Vision Research (17) and were approved by the Tianjin Medical University Animal Ethics Committee (Tianjin, China; approval no. TMUaMEC 2016009; June 6, 2016).

Adult C57BL/6J mice and Smad3-KO mice (18) were employed in the present study (age, 6-8 weeks; weight, $20 \pm 2 \mathrm{~g}$; sex ratio, 76 male and 76 female; $76 \mathrm{KO}$ and $76 \mathrm{WT}$ mice). C57BL/6J mice were purchased from the Institute of Genetics and Developmental Biology at the Chinese Academy of Sciences (Beijing, China). The Smad3-KO mice were provided by Dr Xiao Yang from the Genetic Laboratory of Development and Disease (Institute of Biotechnology, Academy of Military Medical Sciences, Beijing, China). All mice were maintained under a $12 \mathrm{~h}$ light/dark cycle at $22-24^{\circ} \mathrm{C}$ with $45-50 \%$ humidity. Food and water was available ad libitum. The mice were anesthetized by intraperitoneal injections of pentobarbital sodium (70 mg/kg) (19). A 26-gauge hypodermic needle was applied to the right eye to puncture the corresponding central anterior capsule through the corneal limbus, following mydriasis and the administration of topical anesthesia. The depth of the puncture wound was $\sim 25 \%$ of the length of the blade portion of the needle, as previously reported $(17,19)$. The left eye served as an uninjured control. The mice were treated with erythromycin eye ointment and ofloxacin eye drops from $0 \mathrm{~h}$ to 4 weeks post-surgery. Finally, healing mice in the different groups were sacrificed by sevoflurane anesthesia and cervical dislocation to obtain the eyeballs for the subsequent analyses.

Cell culture and in vitro experiments. The HLE-B3 cell line was purchased from American Type Culture Collection (Manassas, VA, USA). The cells were cultured in Dulbecco's modified Eagle's medium/F-12 (HyClone; GE Healthcare Life Sciences, Logan, UT, USA) containing $20 \%$ fetal bovine serum (Gibco; Thermo Fisher Scientific, Inc., Waltham, MA, USA) at $37^{\circ} \mathrm{C}$ with $5 \% \mathrm{CO}_{2}$. The cells were serum-starved overnight and then treated with TGF- $\beta 2(10 \mathrm{ng} / \mathrm{ml} ; \mathrm{R} \& \mathrm{D}$ Systems, Inc., Minneapolis, MN, USA) for $24 \mathrm{~h}$ to measure the ECM component expression levels. In a separate group, the cells were pretreated with $3 \mu \mathrm{M}$ SIS3 (p-Smad3 inhibitor; Sigma-Aldrich; EMD Millipore, Billerica, MA, USA) for $4 \mathrm{~h}$ prior to being treated with TGF- $\beta 2(10 \mathrm{ng} / \mathrm{ml})$. SIS3 was dissolved in $100 \%$ dimethyl sulfoxide (DMSO). DMSO was maintained at a final concentration of $0.1 \%$ in all experiments.

Immunofluorescence staining. Following extraction from the mice, the eyeballs were fixed in $40 \mathrm{~g} / 1$ paraformaldehyde solution for $24 \mathrm{~h}$ at $4^{\circ} \mathrm{C}$. Subsequently, the fixed eyeballs were rinsed with PBS three times for $5 \mathrm{~min}$ each, and the eyeballs were dehydrated in $15 \%$ sucrose solution for $24 \mathrm{~h}$, prior to being placed in $30 \%$ sucrose solution. Each eye was then embedded in optimum cutting temperature compound and sliced into 6- $\mu \mathrm{m}$ sagittal sections with a cryostat (Leica CM 1850; Leica Microsystems GmbH, Wetzlar, Germany). The sections were stored at $-80^{\circ} \mathrm{C}$ until staining.

For the $\alpha$-SMA, E-cadherin, lumican, OPN, fibronectin, HA and collagen type I immunofluorescence localization experiments, the frozen sections were removed from the $-80^{\circ} \mathrm{C}$ freezer, warmed for $30 \mathrm{~min}$ at room temperature and rinsed with PBS three times for $5 \mathrm{~min}$. The sections were then permeabilized with $0.1 \%$ Triton X-100 for $15 \mathrm{~min}$; 5\% bovine serum albumin (BSA; Roche Diagnostics, Basel, Switzerland) was added to the slides for non-specific antigen blocking, and then the slides were incubated for $60 \mathrm{~min}$ at room temperature. The sections were subsequently incubated with primary antibodies specific for $\alpha$-SMA (1:500 dilution; cat. no. ab5694), OPN (1:200 dilution; cat. no. ab8448), lumican (1:200 dilution; cat. no. ab168348), fibronectin (1:500 dilution; cat. no. ab2413) E-cadherin (1:500 dilution; cat. no. ab11512), HA (1:100; cat. no. Sc-7392) and collagen type I (1:500 dilution; cat. no. ab34710) overnight at $4^{\circ} \mathrm{C}$. With the exception of the HA antibody, which was purchased from Santa Cruz Biotechnology, Inc. (Dallas, TX, USA), all of the primary antibodies were purchased from Abcam (Cambridge, MA, USA). The following day, the slides were washed with PBS three times, and they were then incubated with an Alexa Fluor 488-conjugated goat anti-rabbit (A11034), goat anti-rat (A21210), and goat anti-mouse (A11029) secondary antibodies (1:200 dilution; Thermo Fisher Scientific, Inc.) for $60 \mathrm{~min}$ at room temperature. The HLE-B3 cells were cultured in 24-well plates $\left(2 \times 10^{4}\right.$ cells per well $)$ and then treated with TGF- $\beta 2(10 \mathrm{ng} / \mathrm{ml})$ with or without SIS3 $(3 \mu \mathrm{M})$ for $24 \mathrm{~h}$. The cells were rinsed with PBS three times for 5 min each and then fixed with $4 \%$ paraformaldehyde for $30 \mathrm{~min}$. Following washing with PBS, the cells were incubated with 5\% BSA for $30 \mathrm{~min}$ and permeabilized with $0.1 \%$ Triton X-100 for $15 \mathrm{~min}$. The cells were then incubated with primary antibodies specific for fibronectin (dilution 1:200) and E-cadherin (dilution 1:200) overnight at $4^{\circ} \mathrm{C}$. The cells were washed with PBS three times, and then incubated with an Alexa Fluor 488-conjugated goat anti-rabbit and goat anti-mouse secondary antibody (1:200 dilution; Thermo Fisher Scientific, Inc.) for $60 \mathrm{~min}$ at room temperature. Following washing with PBS, the sections and cells were sealed with mounting 
Table I. Primers used for reverse transcription-quantitative polymerase chain reaction analysis.

\begin{tabular}{|c|c|c|}
\hline Gene & Forward primer & Reverse primer \\
\hline$\alpha-\mathrm{SMA}$ & 5'-TCACCATTGGAAACGAACGC-3' & 5'-GCTGTTATAGGTGGTTTCGTGG-3' \\
\hline Snail1 & 5'-CACACGCTGCCTTGTGTCT-3' & 5'-GGTCAGCAAAAGCACGGTT-3' \\
\hline CDH1 & 5'-CAGTTCCGAGGTCTACACCTT-3' & 5'-TGAATCGGGAGTCTTCCGAAAA-3' \\
\hline Slug & 5'-CAGCGAACTGGACACACACA-3' & 5'-ATAGGGCTGTATGCTCCCGAG-3' \\
\hline Twist1 & 5'-GGACAAGCTGAGCAAGATTCA-3' & 5'-CGGAGAAGGCGTAGCTGAG-3' \\
\hline SIP1 & 5'-TCTGTGTAGCCCTGGCTGTC-3' & 5'-GGTGGTCTCGCACTCCTTTA-3' \\
\hline HAS1 & 5'-GCGAGCACTCACGATCATCTT-3' & 5'-GTCCATAGCGATCTGAAGCCA-3' \\
\hline HAS2 & 5'-GTACGGTGCCTTTTTAGCCTC-3' & 5'-TAATCGGGGTTTCAAGGGACT-3' \\
\hline HAS3 & 5'-CCTGGAGCACCGTCGAATG-3' & 5'-CCTTGAGGTTTGGAAAGGCAA-3' \\
\hline Lumican & 5'-TGGGACCACTGTCTTACT-3' & 5'-CTGCTCCAGAACATACTT-3' \\
\hline OPN & 5'-TCCTAGACCCTAAGAGTAA-3' & 5'-TAAGCTAAGAGCCCAAAA-3' \\
\hline Fibronectin & 5'-GAAGACAGATGAGCTTCCCCA-3' & 5'-GGTTGGTGATGAAGGGGGTC-3' \\
\hline Collagen type I & 5'-TTCTGCAGGGTTCCAACGAT-3' & 5'-GCAGGCGAGATGGCTTATTTG-3' \\
\hline GAPDH & 5'-GCAАСТCССАСТСТТСCA-3' & 5'-GTCCAGGGTTTCTTACTCC-3' \\
\hline hGAPDH & 5'-GGAGCGAGATCCCTCCAAAAT-3' & 5'-GGCTGTTGTCATACTTCTCATGG-3' \\
\hline h $\alpha$-SMA & 5'-GTGTTGCCCCTGAAGAGCAT-3' & 5'-GCTGGGACATTGAAAGTCTCA-3' \\
\hline hSnail & 5'-TCGGAAGCCTAACTACAGCGA-3' & 5'-AGATGAGCATTGGCAGCGAG-3' \\
\hline hCDH1 & 5'-CGAGAGCTACACGTTCACGG-3' & 5'-GGGTGTCGAGGGAAAAATAGG-3' \\
\hline hFN1 & 5'-CGGTGGCTGTCAGTCAAAG-3' & 5'-AAACCTCGGCTTCCTCCATAA-3' \\
\hline hCOL1A1 & 5'-GAGGGCCAAGACGAAGACATC-3' & 5'-CAGATCACGTCATCGCACAAC-3' \\
\hline
\end{tabular}

$\alpha$-SMA, $\alpha$-smooth muscle actin; SIP1, SMAD-interacting protein 1; HAS, hyaluronan synthase; OPN, osteopontin; CDH1, E-cadherin; COL1A1, collagen type I $\alpha 1$; FN1, fibronectin 1 .

medium with DAPI (Beijing Solarbio Science \& Technology Co., Ltd., cat. no. S2110). Finally, LEC protein expression was observed with a laser scanning confocal microscope (FV1000; Olympus Corporation, Tokyo, Japan), and images were captured.

Reverse transcription-quantitative polymerase chain reaction $(R T-q P C R)$ analysis. Total RNA was extracted from the lenses ( $\mathrm{n}=4$ samples per group) with the RNeasy Mini kit in accordance with the manufacturer's protocol (Qiagen $\mathrm{GmbH}$, Hilden, Germany). When the HLE-B3 cells had been subjected to the above treatments, they were lysed in RLT buffer. The total RNA concentration was measured using a NanoDrop 2000 spectrophotometer (Thermo Fisher Scientific, Inc.). A total of $2 \mu \mathrm{g}$ of total RNA was used as a template to synthesize cDNA with M-MLV Reverse Transcriptase kit (Promega Corporation, Madison, WI, USA) according to the manufacturer's protocols. The cDNA was then stored at $-20^{\circ} \mathrm{C}$ for later use. qPCR was performed with SYBR Premix Ex Taq $^{\mathrm{TM}}$ (Takara Biotechnology Co., Ltd., Dalian, China), and the data was collected using an StepOnePlus ${ }^{\mathrm{TM}}$ Real-Time PCR system (Applied Biosystems; Thermo Fisher Scientific, Inc.). The qPCR thermocycling conditions were as follows: $95^{\circ} \mathrm{C}$ for $3 \mathrm{~min}$, followed by 40 cycles at $95^{\circ} \mathrm{C}$ for $12 \mathrm{sec}$ and $62^{\circ} \mathrm{C}$ for $40 \mathrm{sec}$, a final extension step of $95^{\circ} \mathrm{C}$ for $15 \mathrm{sec}, 60^{\circ} \mathrm{C}$ for $1 \mathrm{~min}, 95^{\circ} \mathrm{C}$ for $15 \mathrm{sec}$. The relative expression levels of genes were detected and GAPDH served as an internal control. The primer sequences are presented in Table I. The data were calculated by the $2^{-\Delta \Delta \mathrm{Cq}}$ method (20).
Western blot analysis. The lenses ( $\mathrm{n}=4$ per group) from each group were collected and homogenized in lysis buffer (Beyotime Institute of Biotechnology, Haimen, China) containing a protease and phosphatase inhibitor cocktail (Biotool, Shanghai, China). The precipitate was removed through centrifugation $\left(12,000 \mathrm{x}\right.$ g for $5 \mathrm{~min}$ at $\left.4^{\circ} \mathrm{C}\right)$, and the protein concentration of the supernatant was measured using a bicinchoninic acid assay kit (Thermo Fisher Scientific, Inc.). The protein sample from the supernatant was then mixed with 5X SDS-PAGE loading buffer and heated for $5 \mathrm{~min}$ at $98^{\circ} \mathrm{C}$. Equal quantities of proteins $(30 \mu \mathrm{g})$ were separated by $8-10 \%$ SDS-PAGE and then transferred onto a PVDF membrane for $2 \mathrm{~h}$ at $300 \mathrm{~mA}$. The membrane was then incubated with $5 \%$ skim milk for $2 \mathrm{~h}$ at room temperature, and then incubated with primary antibodies specific for $\alpha$-SMA (1:2,000 dilution), lumican (1:2,000 dilution), fibronectin (1:10,000 dilution), OPN (1:5,000 dilution) and GAPDH (1:10,000 dilution) in Blotto overnight at $4^{\circ} \mathrm{C}$. Following three washes with PBST, the membrane was incubated with the appropriate secondary antibody (1:20,000; cat. no. ab6721; Abcam) for $2 \mathrm{~h}$ at room temperature. The membrane was then subjected to three washes with PBST, and protein expression was evaluated by chemiluminescence reagent (EMD Millipore) and exposure to chemiluminescent film. The grayscale images of the proteins were analyzed using ImageJ software (v.1.42q; National Institutes of Health, Bethesda, MD, USA).

TUNEL assay. Apoptosis was measured using a TUNEL detection kit (Roche Diagnostics). Briefly, the frozen 

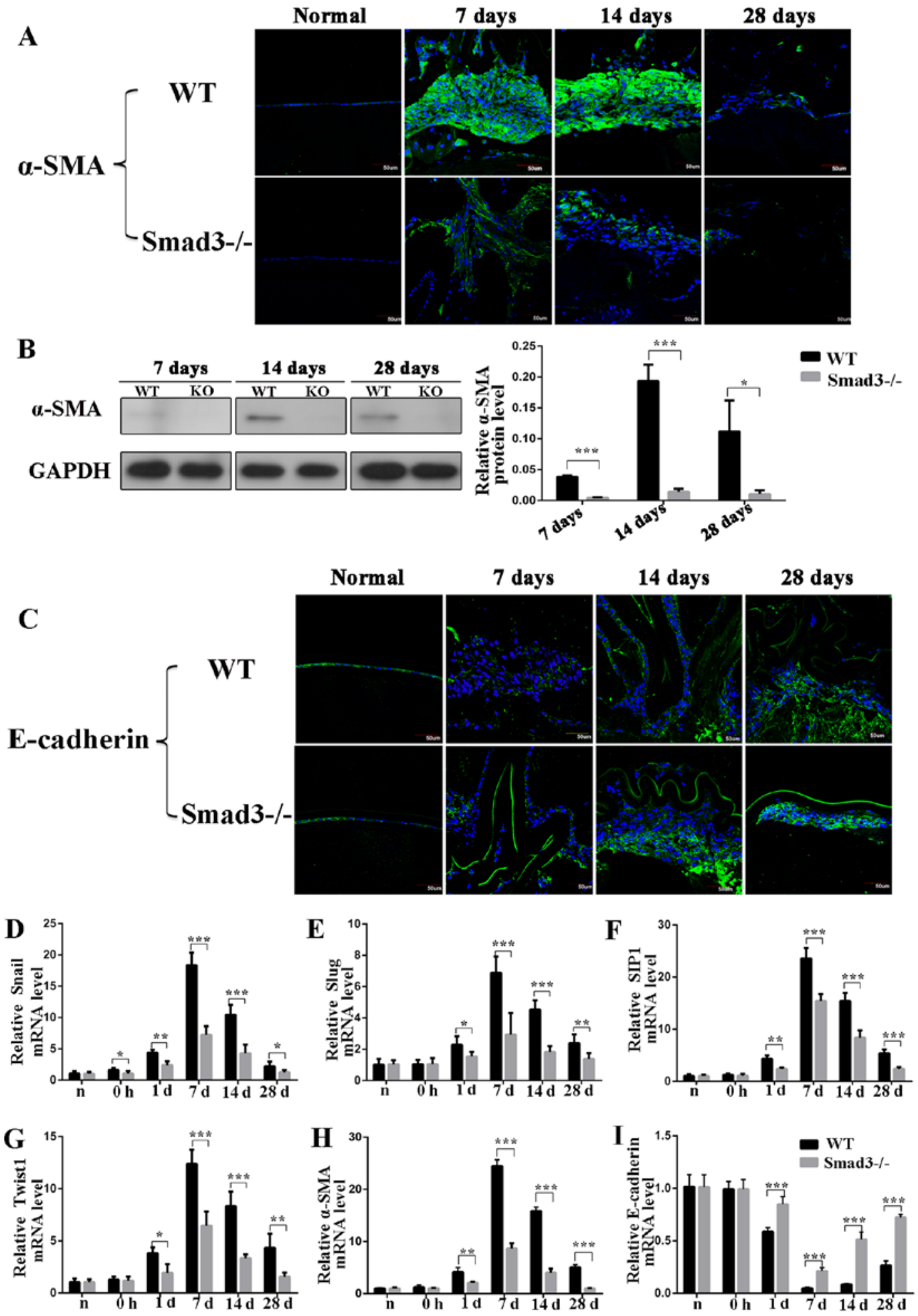

Figure 1. Expression of epithelial-mesenchymal transition biomarkers at different time points following anterior capsular injury. (A) Immunohistochemical analysis of $\alpha$-SMA. (B) Western blot analysis revealed that expression of $\alpha$-SMA was reduced to levels that were $87.5,92.7$ and $90.6 \%$ in Smad3-KO mice of those in WT mice on days 7, 14 and 28, respectively. (C) In situ protein expression of E-cadherin in lens epithelial cells following injury. Transcription factor expression of (D) Snail, (E) Slug, (F) SIP1 and (G) Twist1 in the lens epithelium following injury. (H) Reverse transcription-quantitative polymerase chain reaction analysis revealed that mRNA expression of $\alpha$-SMA in Smad3-KO mice decreased to levels that were 35.5, 25.3 and 20\% of those in WT mice on days 7, 14 and 28 post-injury, respectively. (I) mRNA expression level of E-cadherin at different time points following anterior capsule injury. Scale bar $=50 \mu \mathrm{m}$. ${ }^{*} \mathrm{P}<0.05,{ }^{* *} \mathrm{P}<0.01,{ }^{* * *} \mathrm{P}<0.001$. Smad3, mothers against decapentaplegic 3 ; KO, knockout; WT, wild-type; $\alpha$-SMA, $\alpha$-smooth muscle actin; SIP1, SMAD-interacting protein 1.

sections were removed from storage and air-dried for $30 \mathrm{~min}$ at room temperature. Following washing with PBS, the sections were permeabilized with citrate buffer containing
$0.1 \%$ Triton X-100 for 2 min on ice. DNase I was prepared to treat the positive control samples prior to TUNEL staining. The TUNEL reaction mixture was then added to the sections, 
which were incubated for $1 \mathrm{~h}$ at $37^{\circ} \mathrm{C}$. Finally, the sections were mounted in medium with DAPI, and the resultant images were observed under a fluorescence microscope (DM4000 B LED; Leica Microsystems GmbH). The TUNEL-positive cell nuclei contained green fluorescent particles. For quantitative analysis, five fields in each of the sections from each group were randomly selected and observed at x400 magnification, and the numbers of TUNEL-positive nuclei among 120 LECs were counted.

Statistical analysis. All experimental results are presented as the mean \pm standard deviation of at least three independent experiments. All statistical analyses were performed using SPSS (v.19.0; IBM SPSS, Armonk, NY, USA). Student's t-test was used to assess the differences between two groups. $\mathrm{P}<0.05$ was considered to indicate a statistically significant difference.

\section{Results}

Absence of Smad3 suppresses trauma-induced EMT. To determine whether the absence of Smad3 inhibited the EMT of the lens epithelium following injury, the present study first examined the expression levels of $\alpha$-SMA, a commonly used EMT marker, using immunofluorescence staining. $\alpha-S M A$ was expressed in $\mathrm{Smad}^{-/-}$mice, however, the intensity of immunofluorescence staining intensity in the Smad3 $3^{--}$mice was reduced, compared with that in the WT mice on days 7 , 14 and 28 post-injury (Fig. 1A). Additionally, the expression of $\alpha$-SMA was detected by western blot analysis, the results of which confirmed those of the immunofluorescence experiments. Specially, western blot analysis revealed that the expression levels of $\alpha$-SMA in the Smad3 $3^{-1-}$ mice were reduced by $87.5,92.7$ and $90.6 \%$, compared with those in the Smad $3^{+/+}$mice on days 7,14 and 28 post-injury, respectively $(\mathrm{P}<0.05$; Fig. 1B). The expression of the epithelial marker, E-cadherin, was also detected, the downregulation of which is the most important molecular event in EMT development (21). The Smad3 ${ }^{-/}$mice expressed higher levels of E-cadherin, compared with the WT mice at all time points during EMT. Immunofluorescence staining confirmed that the expression levels of E-cadherin were increased in Smad3 ${ }^{-/}$mice, compared with those in WT mice (Fig. 1C). Additionally, to further confirm the EMT process, the EMT-related transcription factors Snail1, Slug, SIP1 and Twist1 were examined by RT-qPCR analysis. The results showed that the expression of these four transcription factors increased in trauma-induced EMT of LECs and decreased significantly in Smad3-KO mice, compared with WT mice (Fig. 1D-G). As shown in Fig. 1H, the RT-qPCR results revealed that the expression of $\alpha$-SMA in the Smad3-KO mice had decreased to levels that were $35.5,25.3$ and $20 \%$ of those in the WT mice on days 7, 14 and 28 , respectively $(\mathrm{P}<0.001)$. The mRNA expression levels of E-cadherin in the Smad3-KO mice were 4-, 6-, and 2.8-fold higher than those in the WT mice on days 7, 14 and 28 post-surgery, respectively (Fig. 1I).

For the in vitro experiments SIS3 was added to the culture to inhibit the phosphorylation of Smad3. As shown in Fig. 2A, the mRNA expression levels of $\alpha$-SMA and Snail were decreased by 3.6- and 3.7-fold, respectively, and that of E-cadherin was

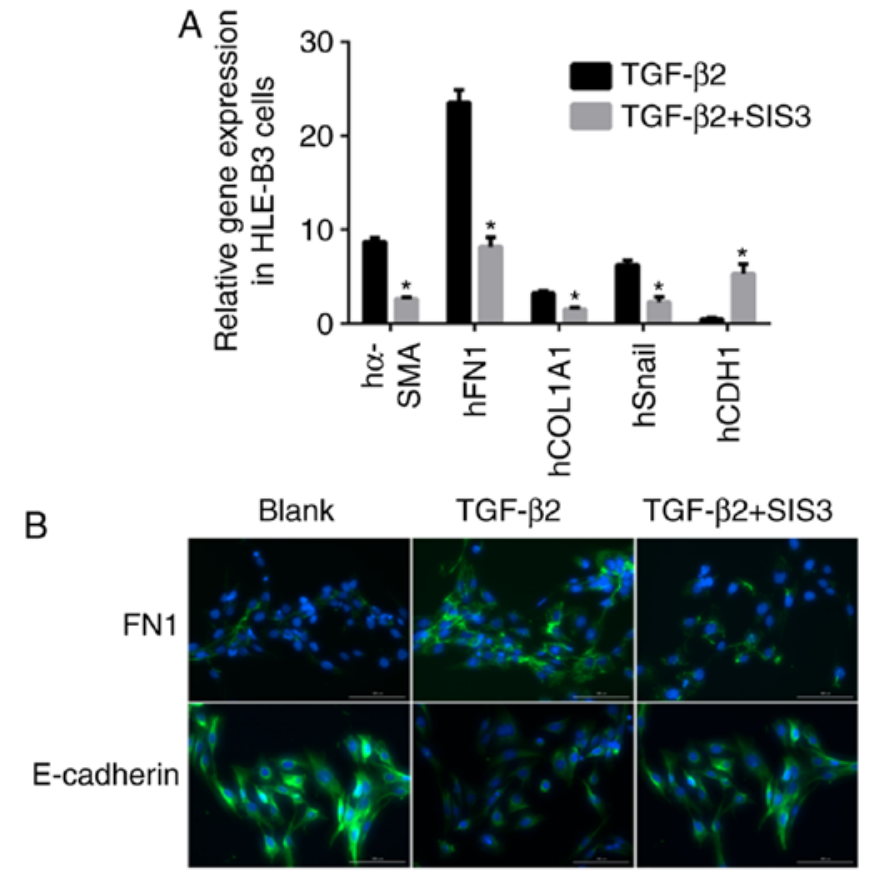

Figure 2. TGF- $\beta / \mathrm{Smad} 3$ pathway involved in EMT to HLE-B3 cells challenged with TGF- $\beta 2$ (10 ng/ml). (A) Gene expression of EMT biomarkers inhibited by SIS3 in HLE-B3 cells. (B) Protein expression of EMT markers in HLE-B3 cells challenged with TGF- $\beta 2(10 \mathrm{ng} / \mathrm{ml})$ following treatment with Smad 3 inhibitor SIS3. Scale bar $=100 \mu \mathrm{m}$. ${ }^{*} \mathrm{P}<0.05$ TGF- $\beta 2+\mathrm{SIS} 3$ group vs. the corresponding TGF- $\beta 2$ group. TGF- $\beta$, transforming growth factor- $\beta$; Smad3, mothers against decapentaplegic 3; EMT, epithelial-mesenchymal transition; $\alpha$-SMA, $\alpha$-smooth muscle actin; FN1, fibronectin 1; COL1A1, collagen type $1 \alpha 1$; CDH1, E-cadherin.

increased by 5.2 -fold in the TGF- $\beta 2+\mathrm{SIS} 3$ group, compared with the TGF- $\beta 2$ group. The immunofluorescence staining confirmed these results (Fig. 2B).

Loss of Smad3 decreases ECM accumulation and suppresses fibrosis during trauma-induced EMT. To examine ECM accumulation during lens epithelium wound healing, the present study detected the expression levels of lumican, OPN, fibronectin, collagen type I and HA (Fig. 3A-a-g). Lumican is an ECM protein belonging to the small leucine-rich proteoglycan family, which binds to cytokines, regulates ECM secretion by mesenchymal cells, and is involved in epithelial cell migration and tissue repair (22). The expression of lumican was examined using RT-qPCR and western blot analyses. As shown in Fig. 3A-a, the mRNA expression of lumican in the Smad3 ${ }^{-1-}$ mice was decreased to levels that were $21.3,11.5$ and $13.2 \%$ of those in the WT mice on days 7, 14 and 28 post-surgery, respectively $(\mathrm{P}<0.001)$. The western blot analysis revealed that the expression of lumican in the Smad3-KO mice decreased to levels that were $52.6,7.9$ and $8.8 \%$ of those in the WT mice on days 7,14 and 28 post-injury, respectively $(\mathrm{P}<0.05)$, which was consistent with the RT-qPCR results (Fig. 3B). Immunofluorescence staining confirmed that the expression of lumican was decreased in the absence of Smad3 (Fig. 3C).

OPN is a glycoprotein, which is secreted into the ECM by several cells and is expressed in PCO and anterior subcapsular cataract (ASC) tissues (23). To obtain additional insight into the role of OPN in the EMT of the lens epithelium and to identify the steps of the EMT that are dependent on Smad3, 

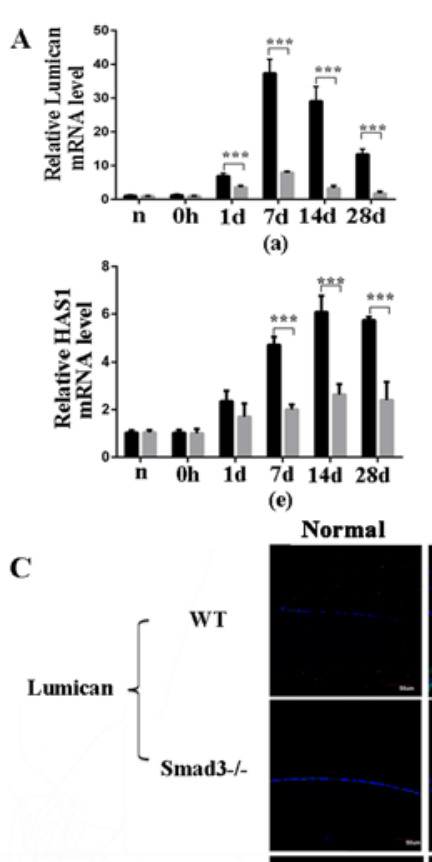

Osteopontin $\left\{\begin{array}{c}\text { WT } \\ \text { Smad3-/- }\end{array}\right.$

G

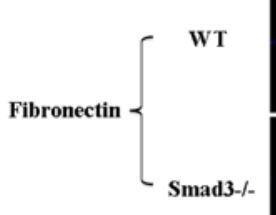

Collagen I $\left\{\begin{array}{l}\text { WT } \\ \text { Smad3-/- }\end{array}\right.$

I

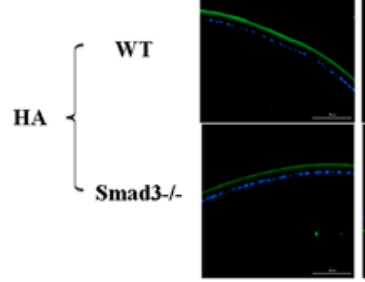

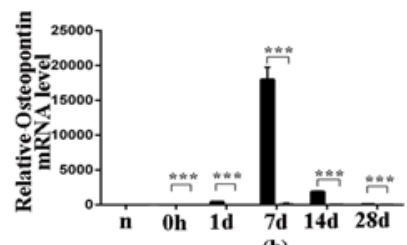

(b)

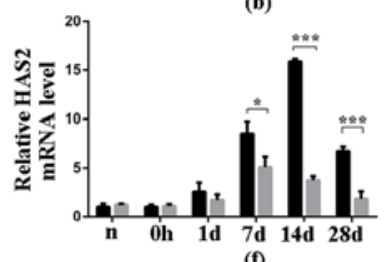

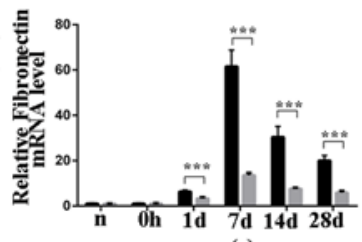

(c)

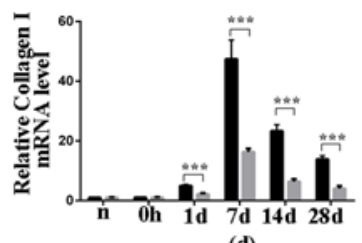

(d)

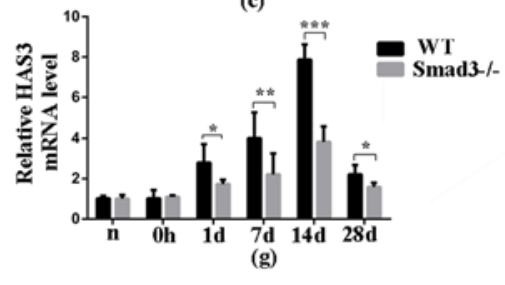

B
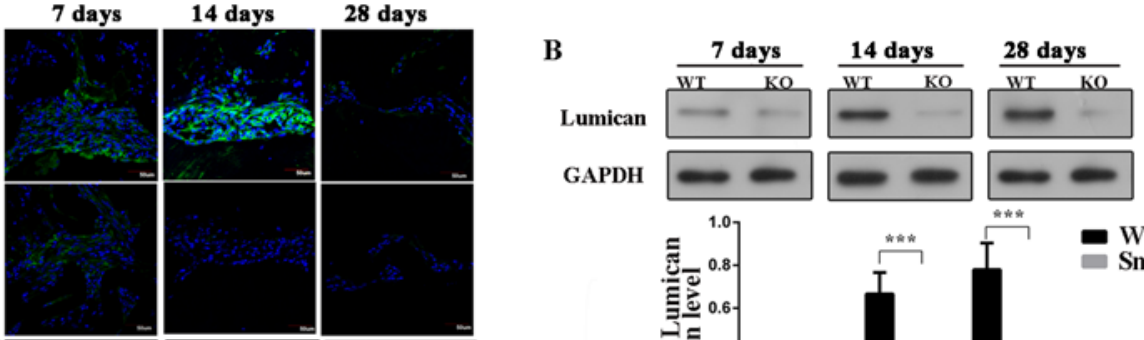

GAPDH

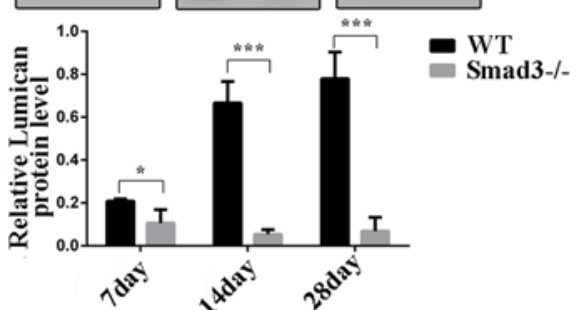

E

GAPDH
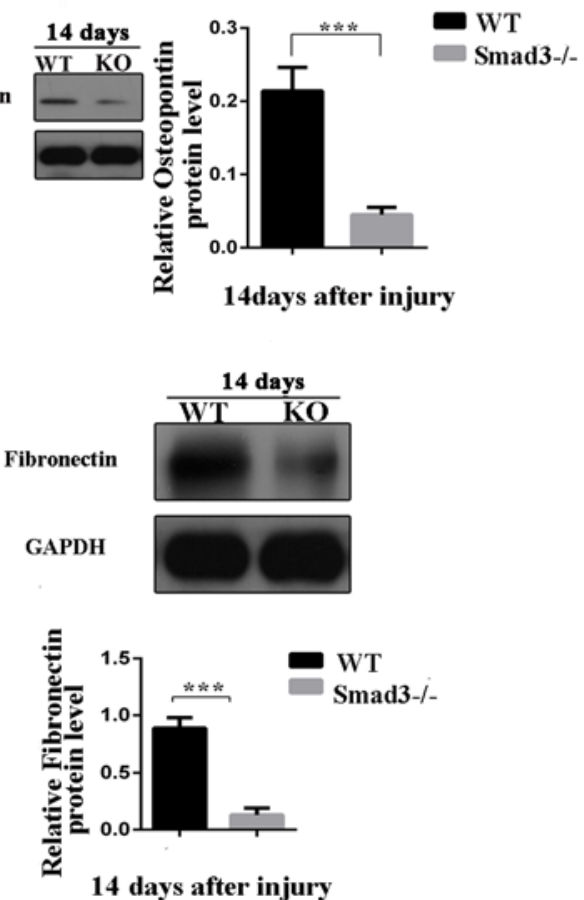

Figure 3. Expression levels of extracellular matrix components at different time points following anterior capsule injury. (A) mRNA expression levels of (a) lumican, (b) oesteopontin, (c) fibronectin, (d) collagen type I and (e-g) HAS in lens epithelial cells following anterior capsule injury. In situ protein expression levels were determined. (B) Western blot and (C) immunofluorescence of lumican; (D) immunofluorescence and (E) western blot of osteopontin; (F) western blot and (G) immunofluorescence of fibronectin. Immunofluorescence of $(\mathrm{H})$ collagen type I and (I) HA in lens epithelial cells following injury. Scale bar=50 or $100 \mu \mathrm{m} .{ }^{*} \mathrm{P}<0.05,{ }^{* *} \mathrm{P}<0.01,{ }^{* * *} \mathrm{P}<0.001$. KO, knockout; WT, wild-type; Smad3, mothers against decapentaplegic 3; HAS, hyaluronan synthase; HA, hyaluronan.

the present study examined OPN expression patterns in WT and KO lenses following injury. The WT LECs exhibited strong immunoreactivity to OPN protein, compared with the Smad3-KO LECs at all time points following injury (Fig. 3D). 

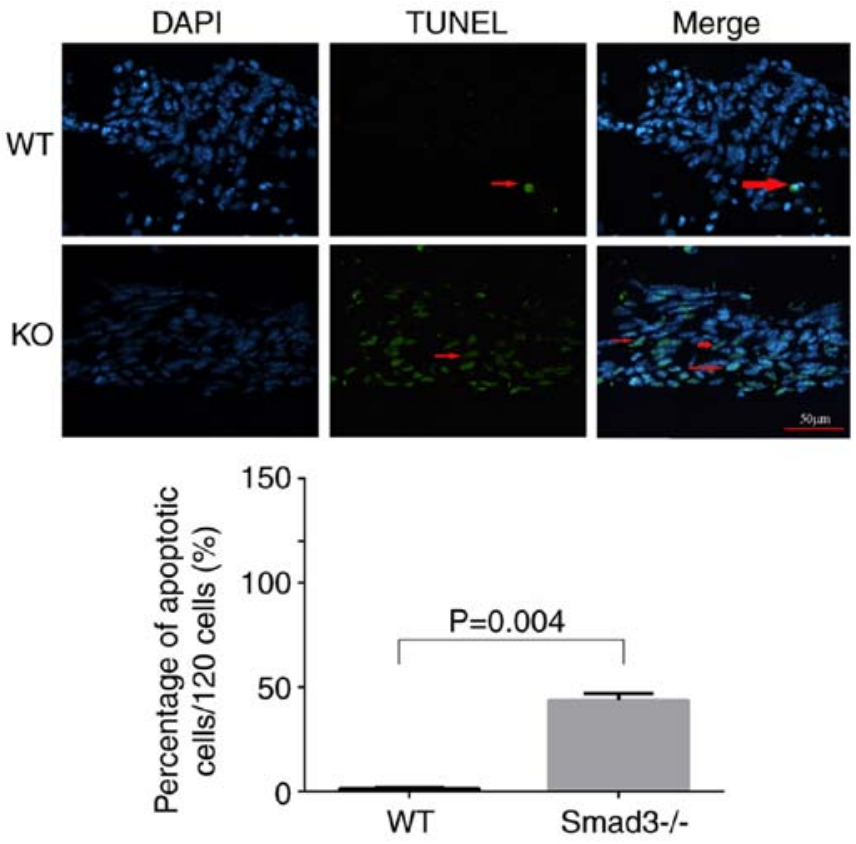

Figure 4. TUNEL staining of apoptotic nuclei in the Smad3-KO and WT lenses. Arrows show TUNEL-positive nuclei (green) around the capsular puncture. The bar graph shows the percentage of apoptotic nuclei per 120 cells in five fields. Smad $3^{-/}$lenses had a significantly higher percentage of apoptotic nuclei $(39.82 \pm 4.4 \%)$, compared with the Smad3 $^{+/+}$lenses $(1.1 \pm 0.44 \%)(\mathrm{P}<0.05)$. Scale bar $=50 \mu \mathrm{m}$. Smad3, mothers against decapentaplegic 3; KO, knockout; WT, wild-type.

As shown in Fig. 3A-b, the mRNA expression of OPN in the Smad3-KO mice was decreased to levels that were 4.4, 2.2 and $5.6 \%$ of those in the WT mice on days 7, 14 and 28 post-surgery, respectively $(\mathrm{P}<0.001)$. The protein expression levels of OPN were measured by western blot analysis on day 14 , and the protein expression of OPN in the Smad3-KO mice was $79.2 \%$ lower than that in the WT mice $(\mathrm{P}<0.001$; Fig. $3 \mathrm{E})$.

Fibronectin is a fibrotic marker and is a major ECM molecule, which is absent in the clear adult lens; however, fibronectin accumulates around LECs in PCO (24). Therefore, the present examined the expression of fibronectin in Smad3-KO and WT mice following puncturing the central anterior lens capsule. The absence of Smad3 decreased the mRNA expression of fibronectin in the Smad3-KO mice to levels that were $22.2,25$ and $29.4 \%$ of those in the WT mice on days 7,14 and 28 post-injury, respectively, as shown in Fig. 3A-c. Additionally, the western blot analysis revealed that the expression of fibronectin in the Smad3-KO was decreased by $85.5 \%$, compared with that in the WT mice on day 14 post-injury (Fig. 3F). Immunoreactivity to fibronectin was compared between the two groups. Fibronectin immunofluorescence intensity in the Smad3-KO group was lower than that in the WT group (Fig. 3G). In the in vitro experiments described above, the mRNA expression levels of fibronectin were 3 -fold lower in the TGF- $\beta 2+$ SIS3 group, compared those in the TGF- $\beta 2$ group (Fig. $2 \mathrm{~A}$ and B), and the immunofluorescence staining confirmed these results.

In the transparent lens, collagen expression is limited to the capsule of the lens; however, abnormal collagen deposition has been identified in ASC plaques and PCO (25). Analysis of the expression of collagen type I was performed using RT-qPCR and immunofluorescence. The mRNA expression of collagen type I in the Smad3-KO mice was decreased to levels that were $65.5,73$ and $71.4 \%$ of those in the WT mice on days 7 , 14 and 28 following anterior capsular injury, respectively (Fig. 3A-d). Immunofluorescence staining confirmed that the expression of collagen type I was decreased in the absence of Smad3 (Fig. $3 \mathrm{H}$ ). In the in vitro experiments, the mRNA expression levels of collagen type I were 2.2-fold lower in the TGF- $\beta 2+$ SIS 3 group than in the TGF- $\beta 2$ group (Fig. $2 \mathrm{~A}$ ).

$\mathrm{HA}$ is found throughout the body, regulates cell proliferation, migration and differentiation, and is key in inflammation and tumorigenesis (26). Hyaluronan synthase (HAS) is an enzyme, which is vital in HA synthesis and exists as one of the following three subtypes: HAS1, HAS2 and HAS3. In the in vivo mouse model, the expression of HA was increased following injury; however, Smad3-KO decreased the expression of HA (Fig. 3I). The expression levels of HAS1, HAS2 and HAS3 were further examined (Fig. 3A-e-g). As shown in Fig. 3A-f, the expression of HAS2 in the Smad3-KO mice was decreased to levels that were $58,23.4$ and $27.6 \%$ of those in the WT mice on days 7, 14 and 28 post-injury, respectively $(\mathrm{P}<0.05)$, and the expression of HAS3 in the Smad3-KO mice was decreased to levels that were $20,48.9$ and $63 \%$ of those in the WT mice on days 7, 14 and 28 post-injury, respectively $(\mathrm{P}<0.05)$ (Fig. 3A-g). However, the expression of HAS1 in the Smad3-KO mice was decreased to levels that were 42.6, 43.3 and $42.1 \%$ of those in the WT mice on days 7, 14 and 28, respectively (Fig. 3A-e).

Taken together, the above data indicated that Smad3 signaling is important in trauma-induced EMT in LECs in vivo and in vitro. The deletion of the Smad3 gene reduced cell transdifferentiation and ECM accumulation, and suppressed fibrosis in trauma-induced EMT in LECs.

Smad3 deficiency induces LEC apoptosis. TUNEL labeling was applied to compare apoptosis levels between the two experimental groups in the present study. LECs around the capsular puncture undergo apoptosis relatively early in the healing process (27). Therefore, lens epithelial cells apoptosis was detected on day 7 using TUNEL staining. The Smad3/- lenses exhibited a significantly higher percentage of TUNEL-positive cells $(39.82 \pm 4.4 \%)$, compared with the $\mathrm{Smad}^{+/+}$lenses $(1.1 \pm 0.44 \%)$ (Fig. 4).

\section{Discussion}

To determine whether Smad3-KO affects trauma-induced EMT in the lens epithelium, differences between the WT and $\mathrm{KO}$ groups were investigated using RT-qPCR analysis, western blot analysis and immunofluorescence staining. It was found that Smad3 deficiency suppressed the EMT. The loss of Smad3 partially inhibited the TGF- $\beta$ signaling pathway in trauma-induced EMT in the lens epithelium. The expression of the EMT marker $\alpha$-SMA, the hallmark of myofibroblasts, was significantly decreased in the Smad3-KO mice, compared with that in the WT mice. The most important change in protein expression that occurs during the EMT is the decrease in the expression of E-cadherin (28). The expression of E-cadherin is downregulated by Snail in the EMT (29). However, the 
expression of E-cadherin, an epithelial marker, was increased in the Smad3-KO mice, indicating that Smad3-KO effectively inhibited TGF- $\beta /$ Smad signaling pathway activity and thus inhibited the EMT. Furthermore, transcription factors Snail1, Slug, SIP1 and Twist1 are involved in the regulation of EMT (29-32), and the data showed that the expression of these transcription factors was increased in trauma-induced EMT and decreased in Smad3-KO mice. The Snail family was mainly involved in this process and consistent with the findings of a previous study (17). The levels of ECM components, including lumican, OPN, fibronectin, HA and collagen type I, were decreased in the Smad3-KO mice, compared with those in the WT mice and were also decreased in the in vitro experiment. These results showed that $\mathrm{Smad} 3$ regulated the production of ECM and were consistent with those of a previous study $(10,16)$. Smad3 may be used as a drug target for the treatment of PCO, as previous studies showed that tranilast and pirfenidone were effective in treating fibrosis by targeting the TGF- $\beta /$ Smad pathway (33-36). Further in vivo experiments in the mouse model used are required to confirm the antifibrotic drug effects. However, in the present study the absence of Smad3 did not completely inhibit trauma-induced EMT in mice in vivo. Capsular injury resulted in the expression of $\alpha$-SMA in the local proliferating cell multilayer, and aberrant ECM deposition, a change reflected by alterations in mRNA and protein expression levels of the marker in Smad3-KO mice; however, the KO lenses exhibited a lower intensity of $\alpha$-SMA staining, compared with that in the WT lenses. This result suggested that Smad3-KO decreased trauma-induced EMT in the lens epithelium and activated non-Smad signaling pathways $(25,37,38)$.

Non-Smad pathways are also important in the EMT. TGF- $\beta$ modulates the EMT by activating protein kinases, including mitogen-activated protein kinase, Ras homolog family member A and phosphoinositide 3-kinase, among others (39-42).

The role of Smad3 in the EMT of epithelial cells remains disputed (8). TGF- $\beta$ activates the Smad3 signaling pathway to promote the occurrence of cataracts (25). Robertson et al utilized recombinant adenoviruses that bind TGF- $\beta$ in the anterior chamber of WT mice and found that these mice developed subcapsular cataracts, however, Smad3-KO mice exhibited delayed cataract development (37). Shirai et al (43) induced a novel ASC model in which the EMT was inhibited in the Smad3-KO mouse lens epithelium by exposing the ocular surface to topical alkali agents. Unexpectedly, the EMT of the LECs was not completely eliminated but was significantly attenuated in Smad3-KO mice. This observation may be associated with the occurrence of a severe inflammatory reaction in the anterior chamber, as inflammatory reactions can activate TGF- $\beta$ and other proinflammatory cytokines, which may result in the activation of Smad3-dependent pathways or non-Smad signaling pathways to regulate EMT (43).

In the present study, ECM deposition was observed in Smad3-KO and WT lenses following injury. The pathologic bases of PCO are residual LEC proliferation, migration and transdifferentiation, collagen deposition, and fibrosis formation following cataract surgery. Abnormal ECM accumulation is common in ASC plaque formation and PCO $(1,44,45)$. However, lower expression levels of lumican, OPN, collagen type I, HA and fibronectin were observed in the Smad3 $3^{-1-}$ lenses, compared with those in the WT lenses, and fewer myofibroblasts were observed in the $\mathrm{Smad}^{-/-}$mice than in the WT mice. These results corresponded with those pertaining to the ECM deposition patterns of the two groups. TGF- $\beta$ is vital in regulating the expression of ECM, and increasing evidence suggest that this process depends on Smad3. Fibrosis is significantly decreased in Smad3-KO wound beds, compared with HT and WT wounds beds (46). In addition, Smad3-KO mice exhibit less TGF- $\beta 2$-induced ocular hypertension and fibronectin deposition in trabecular meshwork cells, compared with WT mice (47). These results indicate that Smad3 is a mediator of the fibrotic response.

Of note, the expression levels of EMT markers and ECM in the $\mathrm{KO}$ group peaked at day 7 post-injury and began to decline at 2 weeks post-injury, but remained higher than the corresponding levels in the normal control group. The EMT markers and ECM components were expressed at low levels at 4 weeks post-surgery. A previous study showed that expression levels of $\alpha$-SMA were highest on day 7 post-surgery (17), which may have been caused by increases in the levels of various cytokines, leading to the development of a high-cytokine 'microenvironment', prompting LEC proliferation and differentiation by 2 weeks post-surgery.

Apoptosis is another characteristic of TGF- $\beta$-induced ASC and PCO $(48,49)$. TGF- $\beta 1 / \mathrm{Smad}^{-/-}$mice have been reported to exhibit a higher percentage of dead cells than WT mice $(25,37)$. In the present study, the number of apoptotic cells was significantly increased in the $\mathrm{Smad}^{-/-}$mice, compared with that in the WT mice. This result was consistent with those of the experiments in which mRNA and protein expression levels were measured, as Smad3 ${ }^{-/-}$mice exhibited lower mRNA and protein expression levels than in WT mice. Smad3-KO may increase apoptosis in trauma-induced EMT in the lens epithelium. The activity of TGF- $\beta$ is generally considered to induce apoptosis. However, the present study found that Smad3-KO increased cell apoptosis, a finding for which the following explanations were suggested: i) a non-canonical TGF- $\beta$ signaling pathway was activated; ii) when arterial injury occurs, levels of Smad3 are high, and TGF- $\beta$ appears to stimulate smooth muscle cell proliferation (50); iii) Smad3 may be required for the survival of intermediate progenitor cells (51), as Smad3 gene-KO disrupts normal cell proliferation, leading to increased apoptosis.

The results of the present study revealed that the deletion of Smad3 inhibited trauma-induced EMT in vivo in the Smad3-KO mouse model and in vitro. As Smad3-KO mice exhibited lower levels of lumican, OPN, collagen type I, HA and fibronectin deposition, compared with those in WT mice, it is possible that signaling pathways independent of Smad3 are activated and that fibrosis is an Smad3-dependent process. Smad3-KO may increase cell death in trauma-induced EMT in the lens epithelium, and Smad3 may be a target for the treatment of PCO and other fibrosis-related diseases.

In conclusion, Smad3-KO decreased trauma-induced EMT in the lens epithelium, but did not completely inhibit EMT. Inhibition of the TGF- $\beta / \mathrm{Smad} 3$ pathway may be clinically desirable for preventing capsular opacification, a potential complication of cataract surgery. 


\section{Acknowledgements}

The authors would like to thank Dr Jinyong Lin and Dr Yuchuan Wang (Department of Pathology of Tianjin Eye Hospital, Tianjin, China), Mr Ming Ying, Mr Peng Hao and Ms Ruifang Han (Tianjin Key Laboratory of Ophthalmology and Visual Science, Tianjin, China) for their technical assistance.

\section{Funding}

This study was supported by grants from the National Natural Science Foundation of China (grant no. 81670837), the Tianjin Research Program of Application Foundation and Advanced Technology (grant no. 13JCYBJC21500), the Tianjin Application Foundation and Advanced Technology Program (15JCYBJC27600), and the Youth Project of Tianjin Applied Basic Research and Cutting-edge Technology Research Programs (grant no. 15JCQNJC45000).

\section{Availability of data and materials}

The datasets used and/or analysed during the current study are available from the corresponding author on reasonable request.

\section{Authors' contributions}

FM performed the experiments, analyzed the data and wrote the paper. XT, XY and JL made substantial contributions to the concept and design of the present study. XY provided the Smad3 knockout mice. JL made substantial contributions to the analysis and interpretation of data. XT and XY revised the paper critically revised the manuscript for important intellectual content. All authors read and approved the final manuscript.

\section{Ethics approval and consent to participate}

All experimental procedures were performed according to the ARVO Declaration on the Use of Animals in Ophthalmic and Vision Research and were approved by the Tianjin Medical University Animal Ethics Committee (approval no. TMUaMEC 2016009; June 6, 2016).

\section{Consent for publication}

Not applicable.

\section{Competing interests}

The authors confirm that they have no competing interests.

\section{References}

1. Awasthi N, Guo S and Wagner BJ: Posterior capsular opacification: A problem reduced but not yet eradicated. Arch Ophthalmol 127: 555-562, 2009.

2. Nibourg LM, Gelens E, Kuijer R, Hooymans JM, van Kooten TG and Koopmans SA: Prevention of posterior capsular opacification. Exp Eye Res 136: 100-115, 2015.

3. Wallentin N, Wickström K and Lundberg C: Effect of cataract surgery on aqueous TGF-beta and lens epithelial cell proliferation. Invest Ophthalmol Vis Sci 39: 1410-1418, 1998.
4. Meacock WR, Spalton DJ and Stanford MR: Role of cytokines in the pathogenesis of posterior capsule opacification. Br J Ophthalmol 84: 332-336, 2000.

5. Lee EH and Joo CK: Role of transforming growth factor-beta in transdifferentiation and fibrosis of lens epithelial cells. Invest Ophthalmol Vis Sci 40: 2025-2032, 1999.

6. Chandler HL, Haeussler DJ Jr, Gemensky-Metzler AJ, Wilkie DA and Lutz EA: Induction of posterior capsule opacification by hyaluronic acid in an ex vivo model. Invest Ophthalmol Vis Sci 53: 1835-1845, 2012.

7. Saika S, Shirai K, Yamanaka O, Miyazaki K, Okada Y, Kitano A Flanders KC, Kon S, Uede T, Kao WW, et al: Loss of osteopontin perturbs the epithelial-mesenchymal transition in an injured mouse lens epithelium. Lab Invest 87: 130-138, 2007.

8. Derynck R and Zhang YE: Smad-dependent and smad-independent pathways in TGF-beta family signalling. Nature 425: 577-584, 2003.

9. Uemura M, Swenson ES, Gaca MD, Giordano FJ, Reiss M and Wells RG: Smad2 and Smad3 play different roles in rat hepatic stellate cell function and alpha-smooth muscle actin organization. Mol Biol Cell 16: 4214-4224, 2005.

10. Li J, Tang X and Chen X: Comparative effects of TGF- $\beta 2 / \mathrm{Smad} 2$ and TGF- $32 / S m a d 3$ signaling pathways on proliferation, migration, and extracellular matrix production in a human lens cell line. Exp Eye Res 92: 173-179, 2011.

11. Flanders KC: Smad3 as a mediator of the fibrotic response. Int J Exp Pathol 85: 47-64, 2004.

12. Brodeur AC, Roberts-Pilgrim AM, Thompson KL, Franklin CL and Phillips CL: Transforming growth factor- $\beta 1 /$ Smad3-independent epithelial-mesenchymal transition in type I collagen glomerulopathy. Int J Nephrol Renovasc Dis 10: 251-259, 2017.

13. Zhao K, He J, Zhang Y, Xu Z, Xiong H, Gong R, Li S, Chen S and He F: Activation of FXR protects against renal fibrosis via suppressing Smad3 expression. Sci Rep 6: 37234, 2016.

14. Cho JY, Doshi A, Rosenthal P, Beppu A, Miller M, Aceves S and Broide D: Smad3-deficient mice have reduced esophageal fibrosis and angiogenesis in a model of egg-induced eosinophilic esophagitis. J Pediatr Gastroenterol Nutr 59: 10-16, 2014.

15. Wormstone IM, Tamiya S, Eldred JA, Lazaridis K, Chantry A, Reddan JR, Anderson I and Duncan G: Characterisation of TGF-beta2 signalling and function in a human lens cell line. Exp Eye Res 78: 705-714, 2004.

16. Li H, Yuan X, Li J and Tang X: Implication of Smad 2 and Smad3 in transforming growth factor- $\beta$-induced posterior capsular opacification of human lens epithelial cells. Curr Eye Res 40: 386-397, 2015.

17. Saika S, Kono-Saika S, Ohnishi Y, Sato M, Muragaki Y, Ooshima A, Flanders KC, Yoo J, Anzano M, Liu CY, et al: Smad3 signaling is required for epithelial-mesenchymal transition of lens epithelium after injury. Am J Pathol 164: 651-663, 2004.

18. Yang X, Letterio JJ, Lechleider RJ, Chen L, Hayman R, Gu H, Roberts AB and Deng C: Targeted disruption of SMAD3 results in impaired mucosal immunity and diminished $\mathrm{T}$ cell responsiveness to TGF-beta. EMBO J 18: 1280-1291, 1999.

19. Xiao W, Chen X, Li W, Ye S, Wang W, Luo L and Liu Y: Quantitative analysis of injury-induced anterior subcapsular cataract in the mouse: A model of lens epithelial cells proliferation and epithelial-mesenchymal transition. Sci Rep 5: 8362, 2015.

20. Livak KJ and Schmittgen TD: Analysis of relative gene expression data using real-time quantitative PCR and the 2(-delta delta $\mathrm{C}(\mathrm{T})$ ) method. Methods 25: 402-408, 2001.

21. Thiery JP, Acloque H, Huang RY and Nieto MA: Epithelial-mesenchymal transitions in development and disease. Cell 139: 871-890, 2009.

22. Yamanaka O, Yuan Y, Coulson-Thomas VJ, Gesteira TF, Call MK, Zhang Y, Zhang J, Chang SH, Xie C, Liu CY, et al: Lumican binds ALK5 to promote epithelium wound healing. PloS One 8: e82730, 2013.

23. Saika S, Miyamoto T, Ishida I, Ohnishi Y and Ooshima A: Osteopontin: A component of matrix in capsular opacification and subcapsular cataract. Invest Ophthalmol Vis Sci 44: 1622-1628, 2003

24. de Jong-Hesse Y, Kampmeier J, Lang GK and Lang GE: Effect of extracellular matrix on proliferation and differentiation of porcine lens epithelial cells. Graefes Arch Clin Exp Ophthalmol 243: 695-700, 2005 . 
25. Banh A, Deschamps PA, Gauldie J, Overbeek PA, Sivak JG and West-Mays JA: Lens-specific expression of TGF-beta induces anterior subcapsular cataract formation in the absence of Smad3. Invest Ophthalmol Vis Sci 47: 3450-3460, 2006.

26. Toole BP, Wight TN and Tammi MI: Hyaluronan-cell interactions in cancer and vascular disease. J Biol Chem 277: 4593-4596, 2002.

27. Saika S, Miyamoto T, Ishida I, Ohnishi Y and Ooshima A: Lens epithelial cell death after cataract surgery. J Cataract Refract Surg 28: 1452-1456, 2002.

28. van Roy F and Berx G: The cell-cell adhesion molecule E-cadherin. Cell Mol Life Sci 65: 3756-3788, 2008.

29. Vincent T, Neve EP, Johnson JR, Kukalev A, Rojo F, Albanell J, Pietras K, Virtanen I, Philipson L, Leopold PL, et al: A SNAIL1-SMAD3/4 transcriptional repressor complex promotes TGF-beta mediated epithelial-mesenchymal transition. Nat Cell Biol 11: 943-950, 2009.

30. Postigo AA, Depp JL, Taylor JJ and Kroll KL: Regulation of Smad signaling through a differential recruitment of coactivators and corepressors by ZEB proteins. EMBO J 22: 2453-2462, 2003.

31. Postigo AA: Opposing functions of ZEB proteins in the regulation of the TGFbeta/BMP signaling pathway. EMBO J 22 : 2443-2452, 2003.

32. Chen ZF and Behringer RR: Twist is required in head mesenchyme for cranial neural tube morphogenesis. Genes Dev 9: 686-699, 1995

33. Choi K, Lee K, Ryu SW, Im M,Kook KH and Choi C: Pirfenidone inhibits transforming growth factor- $\beta 1$-induced fibrogenesis by blocking nuclear translocation of Smads in human retinal pigment epithelial cell line ARPE-19. Mol Vis 18: 1010-1020, 2012.

34. Polydorou C, Mpekris F, Papageorgis P, Voutouri C and Stylianopoulos T: Pirfenidone normalizes the tumor microenvironment to improve chemotherapy. Oncotarget 8: 24506-24517, 2017.

35. Papageorgis P, Polydorou C, Mpekris F, Voutouri C, Agathokleous E, Kapnissi-Christodoulou CP and Stylianopoulos T: Tranilast-induced stress alleviation in solid tumors improves the efficacy of chemo- and nanotherapeutics in a size-independent manner. Sci Rep 7: 46140, 2017.

36. Kaneyama T, Kobayashi S, Aoyagi D and Ehara T: Tranilast modulates fibrosis, epithelial-mesenchymal transition and peritubular capillary injury in unilateral ureteral obstruction rats. Pathology 42: 564-573, 2010.

37. Robertson JV, Nathu Z, Najjar A, Dwivedi D, Gauldie J and West-Mays JA: Adenoviral gene transfer of bioactive TGFbetal to the rodent eye as a novel model for anterior subcapsular cataract. Mol Vis 13: 457-469, 2007.

38. Pervan CL: Smad-independent TGF- $\beta 2$ signaling pathways in human trabecular meshwork cells. Exp Eye Res 158: 137-145, 2017.

39. Secker GA, Shortt AJ, Sampson E, Schwarz QP, Schultz GS and Daniels JT: TGFbeta stimulated re-epithelialisation is regulated by CTGF and Ras/MEK/ERK signalling. Exp Cell Res 314: 131-142, 2008.
40. Bakin AV, Tomlinson AK, Bhowmick NA, Moses HL and Arteaga CL: Phosphatidylinositol 3-kinase function is required for transforming growth factor beta-mediated epithelial to mesenchymal transition and cell migration. J Biol Chem 275: 36803-36810, 2000.

41. Bhowmick NA, Ghiassi M, Bakin A, Aakre M, Lundquist CA, Engel ME, Arteaga CL and Moses HL: Transforming growth factor-beta1 mediates epithelial to mesenchymal transdifferentiation through a RhoA-dependent mechanism. Mol Biol Cell 12: 27-36, 2001.

42. Bhowmick NA, Zent R, Ghiassi M, McDonnell M and Moses HL: Integrin beta 1 signaling is necessary for transforming growth factor-beta activation of p38MAPK and epithelial plasticity. J Biol Chem 276: 46707-46713, 2001.

43. Shirai K, Saika S, Tanaka T, Okada Y, Flanders KC, Ooshima A and Ohnishi Y: A new model of anterior subcapsular cataract: Involvement of TGFbeta/Smad signaling. Mol Vis 12: 681-691, 2006.

44. de Iongh RU, Wederell E, Lovicu FJ and McAvoy JW: Transforming growth factor-beta-induced epithelial-mesenchymal transition in the lens: A model for cataract formation. Cells Tissues Organs 179: 43-55, 2005.

45. Chen X, Xiao W, Chen W, Luo L, Ye S and Liu Y: The epigenetic modifier trichostatin A, a histone deacetylase inhibitor, suppresses proliferation and epithelial-mesenchymal transition of lens epithelial cells. Cell Death Dis 4: e884, 2013.

46. Liu K, Gao Z, Wu X, Zhou G, Zhang WJ, Yang X and Liu W: Knocking out Smad3 favors allogeneic mouse fetal skin development in adult wounds. Wound Repair Regen 22: 265-271, 2014.

47. McDowell CM, Tebow HE, Wordinger RJ and Clark AF Smad3 is necessary for transforming growth factor-beta2 induced ocular hypertension in mice. Exp Eye Res 116: 419-423, 2013.

48. Maruno KA, Lovicu FJ, Chamberlain CG and McAvoy JW: Apoptosis is a feature of TGF beta-induced cataract. Clin Exp Optom 85: 76-82, 2002.

49. Lee JH, Wan XH, Song J, Kang JJ, Chung WS, Lee EH and Kim EK: TGF-beta-induced apoptosis and reduction of Bcl-2 in human lens epithelial cells in vitro. Curr Eye Res 25: 147-153, 2002.

50. Edlin RS, Tsai S, Yamanouchi D, Wang C, Liu B and Kent KC: Characterization of primary and restenotic atherosclerotic plaque from the superficial femoral artery: Potential role of Smad3 in regulation of SMC proliferation. J Vasc Surg 49: 1289-1295, 2009.

51. Tapia-González S, Muñoz MD, Cuartero MI and Sanchez-Capelo A: Smad3 is required for the survival of proliferative intermediate progenitor cells in the dentate gyrus of adult mice. Cell Commun Signal 11: 93, 2013.

This work is licensed under a Creative Commons Attribution-NonCommercial-NoDerivatives 4.0 International (CC BY-NC-ND 4.0) License. 